\title{
Analysis of the Involvement of Hydroxyanthranilate Hydroxycinnamoyltransferase and Caffeoyl-CoA 3-O-Methyltransferase in Phytoalexin Biosynthesis in Oat
}

\author{
Qian Yang, ${ }^{1}$ Hoat Xuan Trinh, ${ }^{1}$ Satoshi Imai, ${ }^{1}$ Atsushi Ishihara, ${ }^{2}$ Liqun Zhang, ${ }^{1}$ Hitoshi Nakayashiki, ${ }^{1}$ \\ Yukio Tosa, ${ }^{1}$ and Shigeyuki Mayama ${ }^{1}$ \\ ${ }^{1}$ Laboratory of Plant Pathology, Graduate School of Science and Technology, Kobe University, Kobe 657-8501, Japan; \\ ${ }^{2}$ Division of Applied Life Sciences, Graduate School of Agriculture, Kyoto University, Kyoto 606-8502, Japan
}

Submitted 15 May 2003. Accepted 6 August 2003.

\begin{abstract}
Two oat genes encoding hydroxycinnamoyl-CoA:hydroxyanthranilate $N$-hydroxycinnamoyltransferase (HHT) and $S$-adenosyl-L-methionine:trans-caffeoyl-CoA 3-O-methyltransferase (CCoAOMT), both of which are possibly involved in the biosynthesis of oat avenanthramide phytoalexins, were cloned and their expression profiles in response to biological stress were studied. Four distinct cDNAs of oat HHT (AsHHT1-4) were isolated with the degenerative polymerase chain reaction method. The enzymatic activity of AsHHT1 expressed in $E$. coli was found using hydroxyanthranilate and hydroxycinnamoyl-CoAs as cosubstrates. Cloned oat CCoAOMT (AsCCoAOMT) encoded a polypeptide of 130 amino acid residues with 77.7 to $80.8 \%$ identities to the CCoAOMT sequences from other plant species. The accumulation of AsHHT1 and AsCCoAOMT transcripts increased concomitantly with phytoalexin accumulation by the treatment of victorin, a specific elicitor in oat lines carrying the $P c-2 / V b$ gene. Pharmacological approaches indicated the involvement of $\mathrm{Ca}^{2+}, \mathrm{NO}$, and protein kinases in the signaling pathways of AsHHT1 and AsCCoAOMT mRNA induction. When oat leaves were inoculated with Puccinia coronata, the mRNA expression of AsHHT1 and AsCCOAOMT increased in both incompatible and compatible interactions but more rapidly in incompatible interaction. Interestingly, however, significant phytoalexin accumulation was observed only in incompatible interaction during the experimental period, suggesting that phytoalexin accumulation may be inhibited in one or more posttranscriptional processes in the compatible interaction.
\end{abstract}

The disease-resistance response of plant cells is composed of a multitude of biochemical events, including the structural reinforcement of cell wall, hypersensitive response (programmed cell death), the development of systemic acquired resistance, and the accumulation of newly produced antifungal chemicals called phytoalexins (Dixon and Paiva 1995; Grayer and Kokubun 2001). We have studied specific resistance mechanisms in the

Corresponding author: S. Mayama; Telephone: +81-78-803-5864; Fax: +81-78-803-5865; E-mail: mayama@kobe-u.ac.jp

Nucleotide sequence data reported are available in the GenBank database under accession numbers AB076979 (AsCCoAOMT), AB076980 (HHT1), AB076981 (HHT2), and AB076982 (HHT3). cultivar-race interactions between oats (Avena sativa L.) and the crown rust fungus (Puccinia coronata $\mathrm{f}$. $\mathrm{sp}$. avenae). In the crown rust-oat system, single gene-mediated rust resistance in the $P$. coronata lines is highly correlated with the production of phytoalexin and the cessation of fungal growth in the primary leaves (Mayama et al. 1981, 1982, 1995a).

Victoria-type resistance to certain races of crown rust is conferred by a single dominant gene of oats, $P c-2$ (Luke et al. 1966). The $P c-2$ oat lines, however, are susceptible to Cochiobolus victoriae because of sensitivity to the fungus-produced host-specific toxin victorin. Victorin sensitivity is also controlled by a single dominant gene, $V b$, in the host plant (Mayama et al. 1995b). Genetic analysis revealed an inability to separate $V b$ from $P c$-2, indicating that the genes conferring victorin sensitivity and controlling crown rust resistance are either very tightly linked or identical (Mayama et al. 1995b). Interestingly, a lower concentration of victorin induces phytoalexin accumulation only in oat lines harboring the $P c-2 / \mathrm{Vb}$ gene and acts, in this context, as a specific elicitor (Mayama et al. 1995b). Thus, the Pc-2/victorin system represents a model system allowing us to study the mechanisms of specific recognition in oats. Using this system, we demonstrated that victorin-induced apoptotic cell death in a sensitive oat line, accompanied by oxidative burst in mitochondria and cellular membrane (Mayama et al. 1986; Tada et al. 2001; Yao et al. 2001, 2002).

Phytoalexins in oats are a set of substituted $N$-hydroxycinnamoyl anthranilates, which are commonly referred to as avenanthramides (Fig. 1) (Mayama et al. 1981; Miyagawa et al. 1995). Hydroxycinnamoyl-CoA:hydroxyanthranilate $N$-hydroxy-cinnamoyltransferase (HHT) catalyzes the N-acylation of hydroxyanthranilate with various hydroxycinnamoyl-CoA as an aroyl-moiety to form a series of avenanthramides in vitro, and the avenanthramides in oats seem to be synthesized by the condensation of corresponding substituted anthranilate and substituted cinnamoyl-CoA thioesters (Fig. 1) (Ishihara et al. 1997, 1999). Oat HHT is biochemically related to hydroxycinnamoyl-/benzoyl-CoA:anthranilate $\quad N$-hydroxycinnamoyl/benzoyltransferase in carnation (Reinhard and Matern 1989; Yang et al. 1997). The acyl-donors, hydroxycinnamoyl-CoAs, are intermediates in the phenylpropanoid pathway (Dixon and Paiva 1995; Douglas 1996; Hahlbrock and Scheel 1989). Avenanthramide B appears to be formed directly from the N-acylation of 5-hydroxyanthranilate with feruloyl-CoA, which is the best acyl-donor for the HHT in vitro (Ishihara et al. 1997). Un- 
der stress conditions, feruloyl-CoA is likely to be formed through the methylation of caffeoyl-CoA catalyzed by elicitorinducible caffeoyl-CoA 3-O-methyltransferase (CCoAOMT) (Fig. 1) (Matern et al. 1995; Pakusch et al. 1989).

Despite the detailed biochemical studies on phytoalexin biosynthesis, the response of the pathway under biological stress remains partly elucidated. Thus, in this paper, we report on the cloning of HHT and CCOAOMT genes from oats and provide detailed analyses on the expression profile of the genes to gain insight into their roles in the defense response to a pathogen in oats.

\section{RESULTS}

\section{Elicitation of phytoalexin production in oat leaves.}

Several kinds of elicitors are capable of inducing the production of avenanthramides in oat leaves (Mayama et al. 1986; Miyagawa et al. 1996). We recently found that crude extracts of the plant growth-promoting rhizobacterium, Pseudomonas fluorescens FPT 9601 (FPT elicitor) also possessed this capacity. Elicitors, including the FPT elicitor, chitosan-hexamer, chitin-hexamer, and victorin $\mathrm{C}$, were examined for phytoalexin induction in oat leaves. At least three times more avenanthramides were produced by treatment with the FPT elicitor than with the other elicitors tested (data not shown). Therefore, we chose FPT elicitor-treated oat leaves to isolate mRNA and, subsequently, constructed a cDNA library.

\section{Cloning of oat HHT and CCoAOMT genes.}

To clone HHT genes from the cDNA library, two degenerate oligonucleotide primers (5'-GCI GCI CAY GTI TTG MGN WS-3' and 5'-GG YTT ICC CCA NCC RAA RTC-3') were synthesized. They were derived from the respective amino acid sequences A-A-H-V-W-R-S and G-F-G-W-G-K-P, both of which are conserved among carnation HCBT ( $N$-hydroxycinnamoyl-benzoyltransferase) and HCBT-like proteins in other plant species. Reverse transcription-polymerase chain reaction (RT-PCR) amplification of mRNA from FPT elicitor-treated oat leaves with these two primers yielded a specific cDNA fragment of $0.4 \mathrm{~kb}$. DNA sequencing and database searches revealed that the cDNA most likely encoded a carnation HCBT-like protein.

Screening of the oat cDNA library $\left(1 \times 10^{5}\right.$ recombinants $)$ with this cDNA probe resulted in the isolation of seven hybridizing clones. Plasmids harboring inserts up to $1.8 \mathrm{~kb}$ were rescued in vivo with the helper phage and found to represent a collection of four distinct cDNAs from 1.2 to 1.8 $\mathrm{kb}$ in length. Three of the four cDNAs, designated AsHHT1 to AsHHT3, encoded isoenzymes that were accordingly named HHT1 to HHT3 (accession numbers AB076980 to AB076982). At both DNA and protein levels, these cDNAs shared 95 to $97 \%$ sequence identity in the coding regions. Sequence diversity was higher in the $5^{\prime}$ and $3^{\prime}$ noncoding regions of the cDNAs. AsHHT4, the fourth cDNA clone was 5 -truncated and had a sequence identity of approximately $75 \%$ with the other three cDNAs. This clone may represent another isoform of the enzyme. The open-reading frames of AsHHT1 to AsHHT3 encoded polypeptides of 441 or 440 amino acid residues. The molecular masses of the polypeptides were calculated as $47.917,47.802$, and $47.782 \mathrm{kDa}$, respectively. The deduced amino acid sequences had 42 to $43 \%$ identity and about $60 \%$ similarity with the carnation HCBT1 and also had almost identical levels of similarity with two putative HCBT proteins derived from the genome sequences of Arabidopsis and sweet potato (Fig. 2). The nucleotide sequences of the oat clones, on the other hand, shared no distinct homology with carnation $H C B T$ and the related genes. The significantly higher $\mathrm{G}+\mathrm{C}$ ratio in oat genes, especially



Fig. 1. A biosynthetic pathway of the avenanthramides. The enzymes involved are 4-coumaroyl-CoA 3-hydroxylase (CCoA3H, EC 1.14.17.-), caffeoylCoA 3-O-methyltransferase (CCoAOMT) and hydroxyanthranilate hydroxycinnamoyltransferase (HHT). 
third position bias, compared with that in the carnation $H C B T$ genes could explain this discrepancy.

Oat CCOAOMT was obtained by random sequencing of cDNA clones screened from the oat cDNA library with subtracted probes. This 622-bp cDNA, designated as AsCCoAOMT (accession number AB076979), encoded an Nterminal truncated polypeptide with sequence similarity to CCoAOMT obtained from other plant species, such as maize (Civardi et al. 1999), alfalfa (Inoue et al. 1998), parsley (Schmitt et al. 1991), grapevine (Busam et al. 1998), and tobacco (Busam et al. 1997). The nucleotide sequence in the coding region of AsCCoAOMT was $88 \%$ identical to a CCoAOMT gene from maize.

Heterologous expression of AtHHT1 in E. coli.

To examine HHT activity, AsHHT1 was heterologously expressed in E. coli. The entire open reading frame of the AsHHT1 cDNA was amplified by PCR, using two synthetic primers, which are described below. The PCR product was digested with $\mathrm{NdeI} / \mathrm{BamHI}$ and was ligated into the multicloning site of the vector pET-22b. The recombinant plasmid desig- nated pET-AsHHT1a was introduced into the expression host E. coli BL21 (DE3). Induction of the vector-encoded T7 lac promoter by adding isopropyl-1-thio- $\beta$-D-galactoside (IPTG) $(1 \mathrm{mM})$ to the culture medium resulted in massive overexpression of a $48-\mathrm{kDa}$ protein in the transformant (Fig. 3, lanes 2 and 4). The $48-\mathrm{kDa}$ protein was mainly found in the supernatant of the bacterial cell lysate (Fig. 3, lane 4). Significant, albeit low-level, HHT activity was detected in crude protein extracts from bacteria transformed with pET-AsHHT1a, which was demonstrated by the formation of avenanthramide B in the presence of 5-hydroxyanthranilic acid and feruloyl-CoA as substrates (Fig. 4A). No HHT activity was detected in the crude protein extracts from $E$. coli transformed with the vectorplasmid (Fig. 4B). These results indicated that AsHHT1 cDNA encoded an active isoform of oat HHT. Low-level activity in the expressed enzyme may be attributed to some posttranslational modifications that are lacking in the bacterium but are required for the high-level catalytic activity of oat HHT. Actually, mouse monoclonal antibody raised against the heterologously expressed AsHHT1 protein allowed us to detect AsHHT1-related proteins in oat plants, with molecular masses

\begin{abstract}
AsHHT1
AtHCBT

IbHCBT

DCHCBT 1

AsHHT 1

AtHCBT

IbHCBT

DCHCBT 1

AsHHT1

AtHCBT

IbHCBT

DCHCBT 1

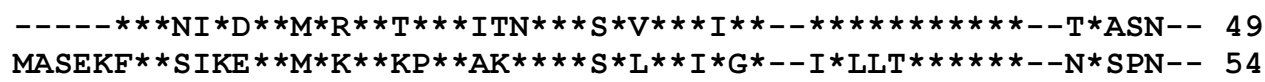

AsHHT1

AtHCBT

IbHCBT

DCHCBT 1

AsHHT1

AtHCBT

IbHCBT

DCHCBT 1

AsHHT1

AtHCBT

IbHCBT

DCHCBT 1

HFINSWSDLCRGVPIAVMPFIDRTL-LRARDPPVPTHPHIEYQPAPAMLGSEE PQALAGK 226

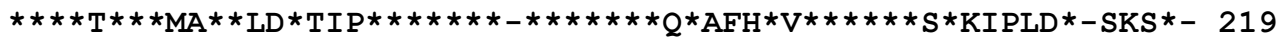

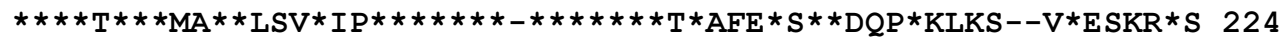
$E \star N * \star \star A R * A K \star L L P \star L E \star V H * \star Y * H * \star P * N * \star Q I K Y S \star S Q F E * F V P S * P N * L L D G K T N * 233$

PESPPTAVDIFKLSRSDLGRLRAQLPTGEGAPRFSTYAVLGAHVWRCASLARGLAPEQPT 286 $\star \star N--\star T * S * \star \star \star T * D Q * V A * K \star K S K E D G N T V S Y * S * E M * A G * \star \star \star S V G K \star \star \star *$ PND $* E * 277$

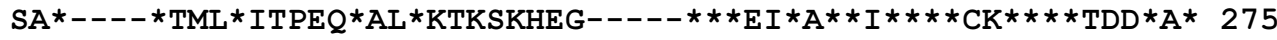

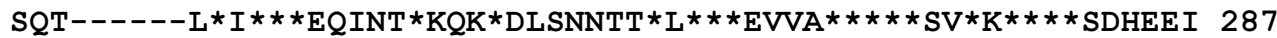

KLYCATDGRQRLT-PTHPDGYFGNVIFTATPLAEAGKVTGS-LADGATTIQDALEKMDDE 344

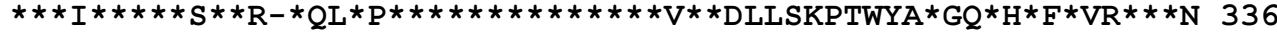

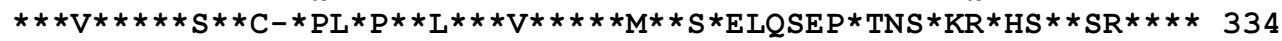

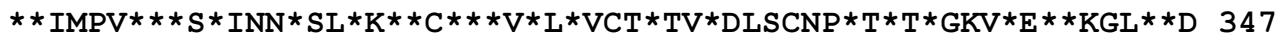

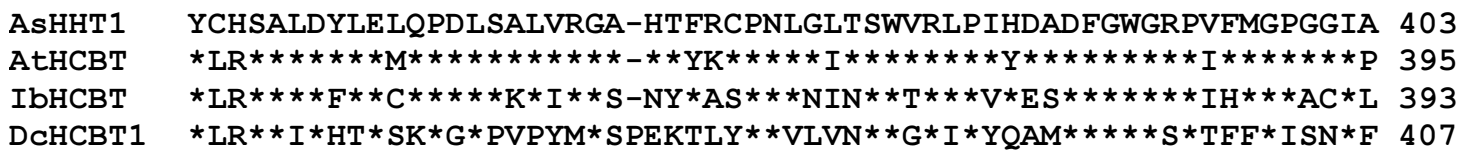

AsHHT1 YEGLAFVLPSANRDGSLSVAISLQAEHMEKFRKMIFDF 441

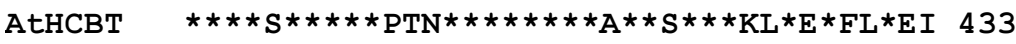

IbHCBT $\quad * \star \star T V Y I * * * \mathrm{P} * \mathrm{~K} * \mathrm{RT} * \star \mathrm{L} * \mathrm{VC} * \mathrm{D} * \star \star \star * \mathrm{PL} * \mathrm{KEFLY} * * 431$

DcHCBT $1 * D * Q C * I I * * R D G * * * M T L * * N * F S S * L S R F K * Y F Y * * 445$

Fig. 2. Clustal alignment of oat hydroxyanthranilate hydroxycinnamoyltransferase (HHT) with carnation HCBT and related plant proteins. The deduced amino acid sequence of AsHHT1 was aligned to those of HCBT-like proteins from Arabidopsis (AtHCBT; accession number NP-199704) and sweet potato (IbHCBT; accession number BAA87043) as well as carnation HCBT1 (DcHCBT1; accession number Z84383). Asterisks indicate amino acid residues identical to those of AsHHT1. Bars indicate gaps introduced to optimize alignment. 
of 28, 39, and $47 \mathrm{kDa}$, by Western blot (immunoblot) analysis (data not shown). The $39-\mathrm{kDa}$ form of HHT protein was constantly and most abundantly found. In contrast, the $47-\mathrm{kDa}$ form, which likely corresponded to a full length of AsHHT1, was increased in amount only upon stress treatments (data not shown). This might suggest that HHT protein was synthesized in a $47-\mathrm{kDa}$ form and subsequently processed to be active forms (39 or $28 \mathrm{kDa}$ ) in planta.

\section{Expression of HHT and CCoAOMT genes in infected oat leaves.}

Previous investigations have revealed that phytoalexin production is correlated with resistance gene expression and is effective in preventing the development of crown rust fungus in oat leaves (Mayama et al. 1982). In this study, we examined the expression patterns of HHT and CCOAOMT genes during compatible and incompatible interactions between oat and the crown rust fungus. Similar expression patterns upon rust infection were observed with AsHHT1 and AsCCOAOMT. The mRNA level of AsHHT1 and AsCCOAOMT in oat leaves was undetectable before inoculation (Fig. 5A). At $6 \mathrm{~h}$ after inoculation, low-level induction of AsHHT1 and AsCCOAOMT expression was detected in both compatible and incompatible interactions, probably because of mechanical stress exerted by talc during inoculation (discussed below). In incompatible interactions, the AsHHT1 mRNA level increased significantly at $12 \mathrm{~h}$, reached a maximum at $36 \mathrm{~h}$, and then returned to the basal level. Similarly, the mRNA level of AsCCoAOMT increased visibly at $12 \mathrm{~h}$, reached a maximum at $24 \mathrm{~h}$, and then decreased thereafter. In compatible interactions, low levels of AsHHT1 mRNA were maintained $18 \mathrm{~h}$ after inoculation, and a significant increase was detected later at $24 \mathrm{~h}$ after inoculation and remained at high levels thereafter. A very similar expression pattern to AsHHT1 mRNA was observed with AsCCOAOMT in compatible interactions. On the other hand, the expression pattern of AsHHT4 upon rust infection was a little different from those of the other two genes (Fig. 5B). AsHHT4 mRNA was detectable even in healthy leaves, and the expression pattern did not differ much between compatible and incompatible interactions, except that AsHHT4 transcription was upregulated 12 and $48 \mathrm{hr}$ after inoculation slightly more in incompatible interaction than in compatible interactions. Constitutive expression of AsHHT4 in healthy oat leaves suggested that this gene may be involved in the synthesis of one or more chemical compounds other than phytoalexins.

Phytoalexin accumulation during compatible and incompatible oat-rust interactions was also measured (Fig. 5C). In in-

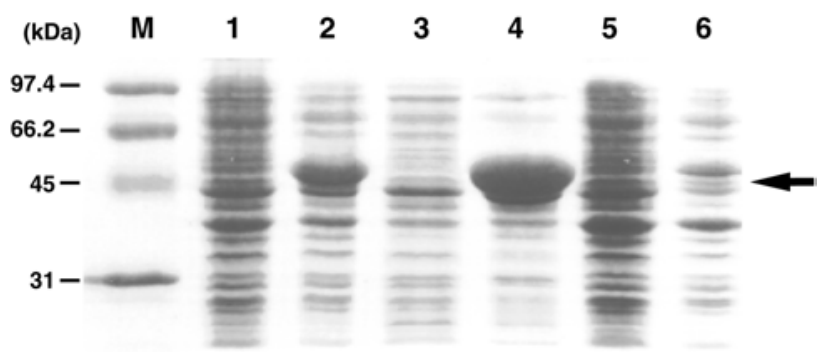

Fig. 3. Sodium dodecyl sulfate-polyacrylamide gel electrophoresis (SDSPAGE) examination of oat HHT1 expressed in Escherichia coli. Proteins $(10 \mu \mathrm{g})$ from the total cell lysates (lanes 1 and 2$)$, cell lysis supernatants (lanes 3 and 4), and lysis pellets (lanes 5 and 6) of noninduced transformed E. coli harboring pET-AsHHTIa (lanes 1, 3, and 5) or of the isopropyl-1-thio- $\beta$-D-galactoside-induced $E$. coli (lanes 2, 4, and 6) were separated on $12 \%$ SDS-PAGE and were subsequently stained with Coomassie blue. Molecular mass markers (lane M) are indicated in the left margin, and the position of the HHT1 polypeptide is indicated by an arrow in the right margin. compatible interaction, the expression pattern of AsHHT1 and AsCCoAOMT genes was correlated with phytoalexin accumulation, suggesting the active participation of AsHHT1 and AsCCoAOMT in phytoalexin production to restrict fungal growth. Intriguingly, only very low levels of phytoalexin accumulation were observed in compatible interactions throughout the experiment, despite the fact that the expression of both AsHHT1 and AsCCoAOMT genes was strongly induced at 24 $\mathrm{h}$ and thereafter (Fig. 5A and C). These results suggested that phytoalexin accumulation in compatible interactions was blocked in one or more posttranscriptional processes, including the translation or modification, or both, of proteins, the lack of substrates in the cell, and the degradation of synthesized phytoalexins.

\section{Induction of HHT and CCoAOMT gene expression by victorin and signaling pathways involved.}

Victorin induced phytoalexin accumulation specifically in oat lines carrying the $P c 2 / V b$ gene, such as 'Iowa $\times 469$ ' (Mayama et al. 1986). RNA gel blot analysis revealed that victorin concomitantly induced the accumulation of AsHHT1 and AsCCoAOMT transcripts in the victorin-treated leaves (Fig. 6). A considerable increase in AsHHT1 mRNA accumulation was seen $6 \mathrm{~h}$ after victorin treatment, which increased significantly at $12 \mathrm{~h}$, reached a maximum at $18 \mathrm{~h}$, and remained at
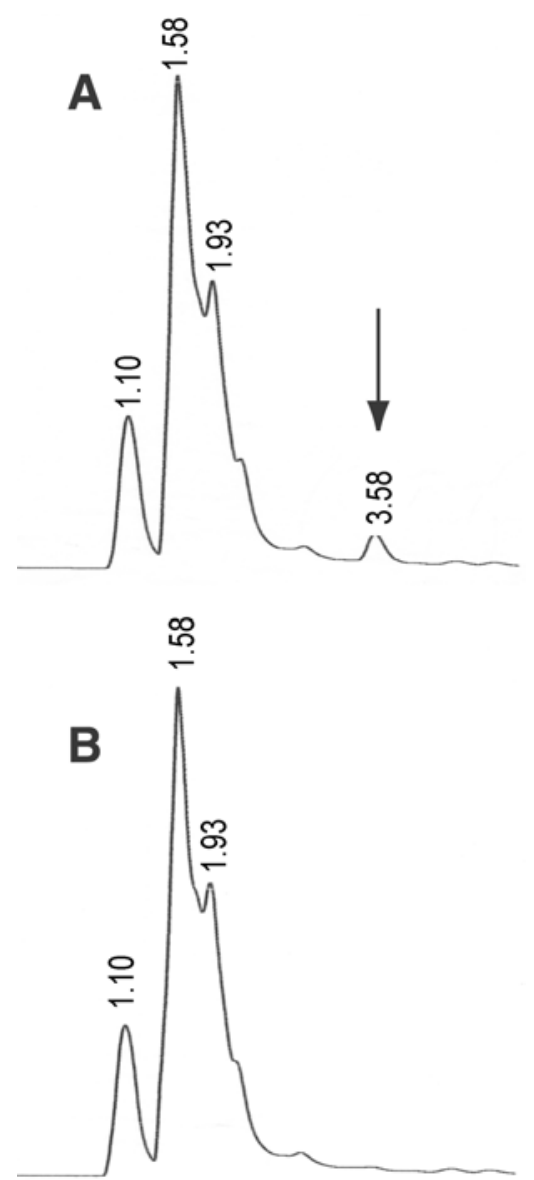

Fig. 4. Reversed-phase high-pressure liquid chromatography (HPLC) separation of product formed by crude protein extracts of Escherichia coli expressing HHT1. Hydroxyanthranilate hydroxycinnamoyltransferase (HHT) activity was assayed with 5-hydroxyanthranilic acid and feruloyl-CoA, and the product avenanthramide B (indicated by an arrow) was detected by HPLC. A, Crude protein extracts from E. coli harboring pET-AsHHT1a catalyzed the formation of avenanthramide B. B, Control incubation with crude protein extracts from $E$. coli transformed with vector $\mathrm{pET}-22 \mathrm{~b}$. 
high levels until $24 \mathrm{~h}$ (Fig. 6). In contrast, only a low level of AsHHT1 mRNA was detectable in the water-treated control leaves. Victorin induced the expression of the AsCCoAOMT gene even more prominently. The abundance of AsCCoAOMT transcripts reached maximum as early as $6 \mathrm{~h}$ and remained at high levels up to $24 \mathrm{~h}$ in the victorin-treated leaves (Fig. 6). Transient and low-level increases in AsCCoAOMT transcripts, on the other hand, were observed in the control leaves treated with water. This is plausible due to an induction effect caused by wounding when the leaves were peeled. Victorin did not induce phytoalexin accumulation in the oat line 'Iowa×424', which carried no $P c 2 / \mathrm{Vb}$ gene (Mayama et al. 1986, 1995b).
Correspondingly, no significant increase in either AsHHT1 or AsCCOAOMT mRNA accumulation was observed in the victorin-treated 'Iowa×424' leaves (data not shown).

Previous pharmacological research has indicated that there is victorin-induced phytoalexin accumulation in oats via signal mediators such as calcium influx, calmodulin activation, kinases, and reactive oxygen species (Tada et al. 2000). In this study, we examined which mediators were actually involved in the transcriptional induction of AsHHT1 and AsCCoAOMT genes. Similar to victorin, calcium ionophore A23187 induced AsHHT1 and AsCCoAOMT gene expression, as well as phytoalexin accumulation (Fig. 7) (Ishihara et al. 1996). However,

\section{A}

compatible incompatible

$\begin{array}{llllllllllllll}0 & 6 & 12 & 18 & 24 & 36 & 48 & 6 & 12 & 18 & 24 & 36 & 48 & (\mathrm{~h})\end{array}$

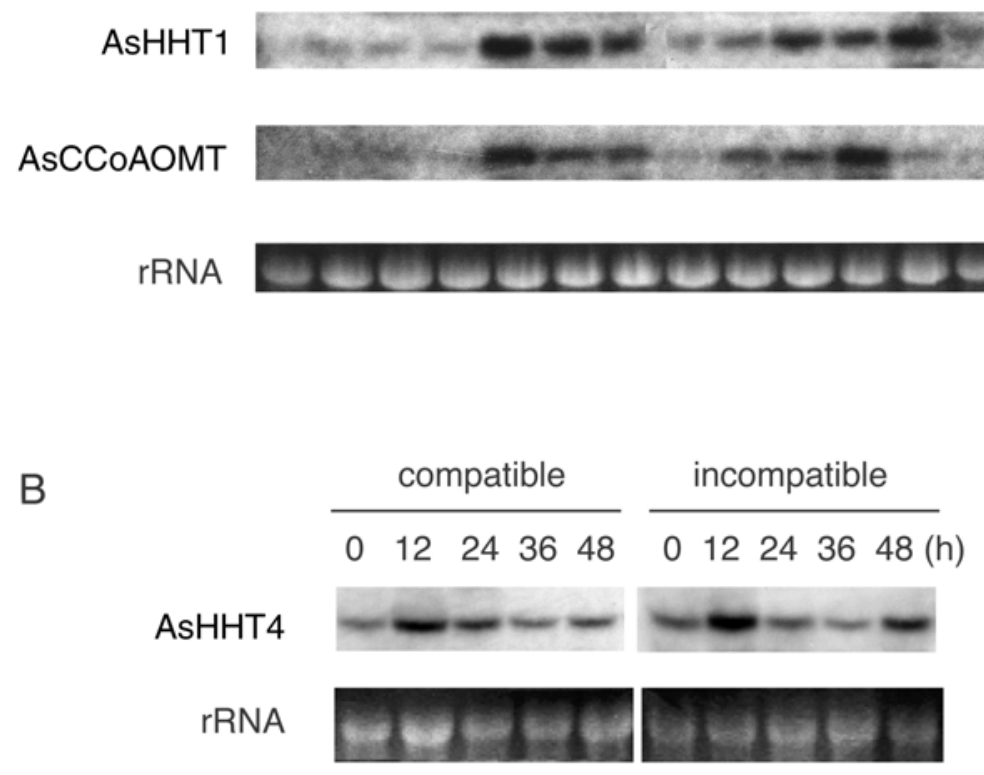

C

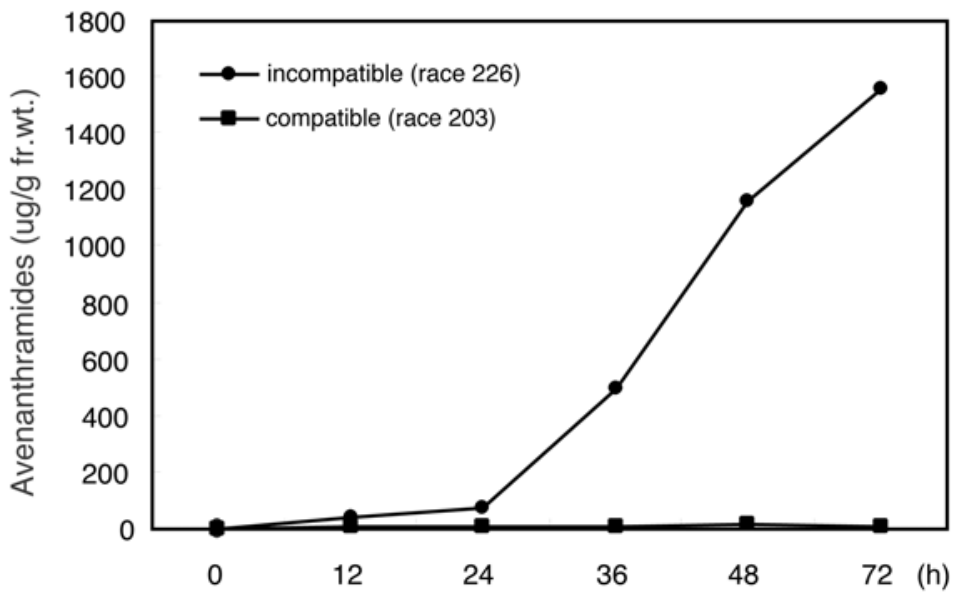

Fig. 5. Expression of the A, AsHHT1, AsCCoAOMT, and B, AsHHT4 genes and $\mathbf{C}$, phytoalexin accumulation in oat leaves during compatible and incompatible interactions with the crown rust fungus. Oat cultivar Shokan 1 and oat races 203 (compatible) and 226 (incompatible) were used in A and C. Rust race 226 and oat cultivars Shokan1 (resistant) and PC-38 (susceptible) were employed in B. A and B, Expression patterns of HHT and CCoAOMT were detected by Northern analysis. C, Accumulation of the phytoalexin avenanthramides was monitored by high-pressure liquid chromatography, using reversed-phase column (Hitachi gel \#3056, $4 \times 150 \mathrm{~mm}$ ) eluted with $29 \%$ acetonitrile in distilled water containing $1 \%$ phosphoric acid. The amount of phytoalexin accumulation was estimated by comparing the peak area for UV absorbance at $340 \mathrm{~nm}$ with a standard curve made with authentic synthesized avenanthraimde A. 
the accumulation of AsHHT1 and AsCCoAOMT mRNA induced by victorin was completely negated by cotreatment of oat leaves with either nifedipine (calcium channel blocker) or trifluoperazine (calmodulin antagonist) (Fig. 7), and the phytoalexin accumulation was also completely inhibited (Tada et al. 2001). These results indicated that calcium influx and subsequent calmodulin activation were critical events in activating the phytoalexin biosynthesis pathway in oats. The inhibitors of protein kinases, K252a and KT5823, moderately reduced the accumulation of AsHHT1 and AsCCoAOMT mRNA induced by victorin. Similarly, the administration of L-NMMA (NO synthase inhibitor) and cPTIO (NO scavenger) significantly but not completely inhibited gene expression of AsHHT1 and AsCCoAOMT induced by victorin. Interestingly, previous studies showed that kinase and NO inhibitors at the concentrations used here inhibited victorin-induced phytoalexin accumulation almost completely in the sensitive oat line. This indicated that the kinase and NO inhibitors also affected one or more processes other than the transcriptional activation of

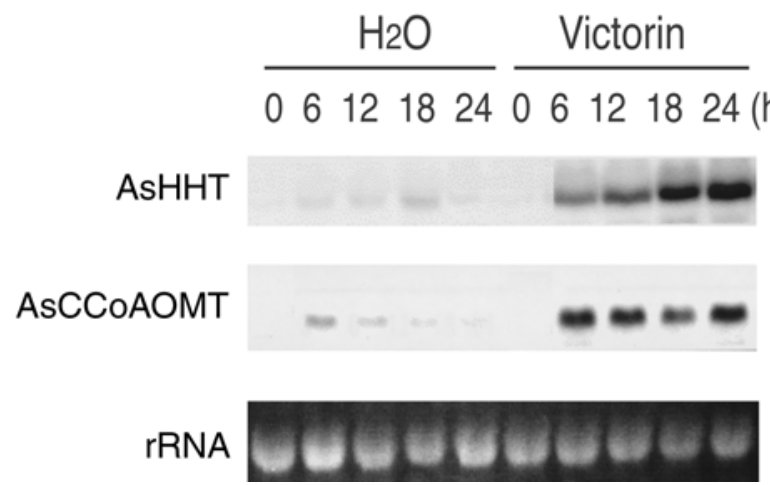

Fig. 6. Induced expression of the AsHHT1 and AsCCoAOMT genes in oat leaves by victorin. Peeled leaves of oat cv. Iowa 469 were treated with victorin $\mathrm{C}(0.25 \mathrm{ng} / \mathrm{ml})$ or water.
AsHHT1 and AsCCoAOMT genes in the phytoalexin biosynthesis pathway. Nevertheless, the inhibitors that blocked phytoalexin accumulation generally negated transcriptional activation of the AsHHT1 and AsCCoAOMT genes, suggesting that mass production of phytoalexin in response to elicitors was regulated, at least partly, by the transcriptonal activation of the phytoalexin biosynthesis genes.

\section{DISCUSSION}

The synthesis of avenanthramides in oats formally requires the provision of hydroxycinnamoyl-CoAs as acyl donors (Fig. 1), which are intermediates in the phenylpropanoid pathway (Dixon and Paiva 1995; Douglas 1996; Hahlbrock and Scheel 1989). Dual pathways have been proposed for the formation of hydroxycinnamoyl-CoAs, except 4-coumaroyl-CoA, which is formed directly from coumaric acid by 4-coumaric acid CoAligase (Dixon and Paiva 1995; Ye et al. 1994). Oat avenanthramides chemically resemble dianthramides, the phytoalexins identified in carnation (Niemann 1993). The amide-forming enzymes in oats and carnatinon, i.e., HHT and HCBT, use anthranilate as an acyl receptor and accept a variety of aromatic acyl-CoAs as acyl donors. However, carnation HCBT exhibited narrow substrate specificity for anthranilate (Reinhard and Matern 1989; Yang et al. 1997). Anthranilate-derived phytoalexins are supposed to play an important role in the disease resistance of oats to crown rust and of carnation to Fusarium wilt. This is based on fungi toxicity and the site, timing, and concentration of phytoalexin accumulation in infected tissue in relation to the restriction of fungal development (Mayama et al. 1982, 1995a; Miyagawa et al. 1995; Niemann 1994). Furthermore, phytoalexin production in oat leaves was directly proportional to the degree of resistance expression against crown rust (Mayama et al. 1982). Thus, oat HHT is likely to play a pivotal role in resistance of oats to disease.

Interestingly, our results indicated that phytoalexin accumulation upon pathogen infection did not merely depend on the

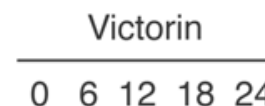

\section{AsHHT \\ AsCCOAOMT}

rRNA
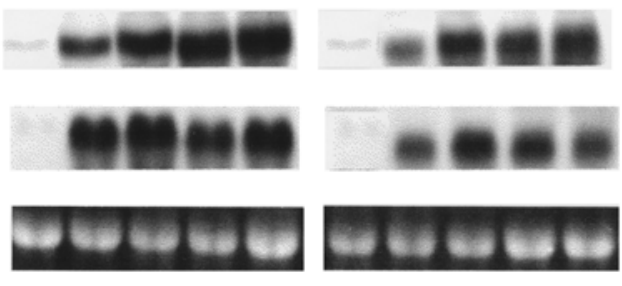
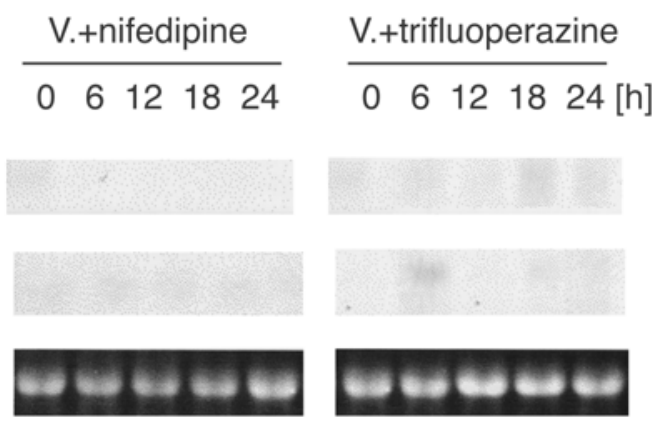

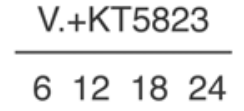

AsHHT

AsCCOAOMT

rRNA
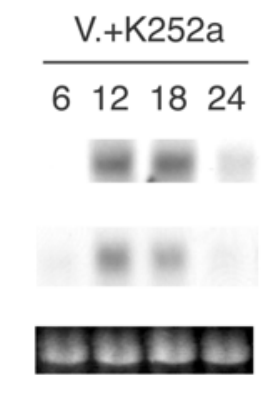



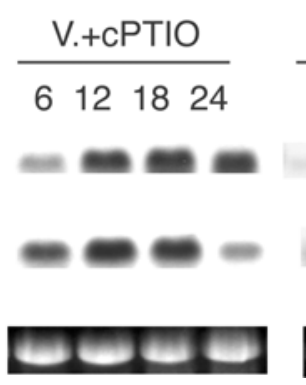

Fig. 7. Expression pattern of the AsHHT1 and AsCCoAOMT genes in oat leaves treated with elicitors and inhibitors. Peeled leaves of oat cv. Iowax469 were cotreated with victorin $\mathrm{C}(0.25 \mathrm{ng} / \mathrm{ml})$ and an inhibitor. A23187 (a calcium ionophore, $10 \mu \mathrm{M}$ ) was used alone without victorin C. The concentrations of the inhibitors used were as follows: $5 \mathrm{mM}$ nifedipine (a calcium channel blocker), $500 \mu \mathrm{M}$ trifluoperazine (a calmodulin antagonist), $5 \mu \mathrm{M}$ K-252a (serine-threonine protein kinase inhibitor), $10 \mu \mathrm{M}$ KT5823 (protein kinase G inhibitor), 2 mM L-NMMA (NO synthase inhibitor), and 500 $\mu$ M cPTIO (NO scavenger). 
transcriptional activation of phytoalexin-biosynthetic genes such as oat HHT and CCoAOMT. Increased expression of AsHHT1 and AsCCoAOMT in 'Shokan 1' oat leaves inoculated with compatible race 203 of $P$. coronata did not result in phytoalexin accumulation to significant levels (Fig. 5) (Mayama et al. 1982). This indicated that the phytoalexins were either degraded or not synthesized in oat cells infected with the compatible rust fungus. Since oat cells remain alive until the later stage of infection in the case of compatible obligate parasites (Yao et al. 2002), phytoalexins may be catalyzed for simultaneous degradation as a metabolic response for removing toxic compounds from the cell. In incompatible interactions, on the other hand, infected oat cells undergo the hypersensitive dying process at the early stage of infection (Mayama et al. 2001; Tada et al. 2001), and deposited phytoalexins may not be metabolized further, and therefore, may not accumulate at these sites. Alternatively, the phytoalexins were not synthesized due to metabolic derangement of the oat cell caused by successful fungal infection (e.g., fungal toxic compounds). The substrates of HHT and CCoAOMT that are required to fully activate the enzymes might not be sufficiently supplied or some posttranscriptional modifications of HHT and CCoAOMT may have interfered. We cannot rule out, however, the possibility that there may be other oat HHT and CCoAOMT isoforms that are involved in phytoalexin production upon pathogen infection in planta. With the help of in situ hybridization and immunological techniques, more precise timing and localization of proteins and gene expression can be determined in infected plants, leading to a better understanding of the function of phytoalexin accumulation in the resistance of oats to disease.

The sensitivity of oats to victorin $\mathrm{C}$ is determined by a single dominant gene, $V b$, which is closely linked with, or the same as, the $P c-2$ gene for resistance to certain races of the crown rust fungus (Mayama et al. 1995b). The $P c-2 / V b$ gene product presumably functions at an early stage of the signal transduction pathway, prior to the activation of phytoalexin biosynthesisrelated genes. The specific recognition between the $P c-2 / V b$ gene product and victorin is considered to initiate a series of defense responses in oats. These include apoptotic cell death with characteristic chromatin condensation, DNA cleavage, and DNA laddering (Tada et al. 2001; Yao et al. 2001), as well as the activation of defense related genes, such as pathogenesis-related protein genes and phytoalexin-biosynthetic genes (Mayama et al. 2001). Interestingly, pharmacological studies have suggested that the downstream events induced by victorin seemed to occur via various signaling pathways. For example, kinases have been suggested to be involved in chromatin condensation but not in nuclear DNA laddering (Yao et al. 2001). The present study revealed that the kinase and NO inhibitors were only partly able to down regulate the transcription activation of AsHHT1 and AsCCoAOMT genes, whereas they almost completely abolished victorin-induced phytoalexin accumulation but had no effect on nuclear DNA laddering (Tada et al. 2000, 2001). Furthermore, an antagonist of calmodulin, trifluoperazine, inhibited both phytoalexin accumulation and its related gene activation but not DNA laddering induced by victorin (Tada et al. 2000, 2001). It is noteworthy that the inhibitors of calcium influx, such as nifedipine and ruthenium red, commonly interfered with all the downstream events we examined (Tada et al. 2000, 2001). This may suggest that calcium influx is the initial and crucial event that activates the signal transduction triggered by victorin in sensitive oat lines. The recognition of victorin by the $P c-2 / V b$ gene product may open calcium channels in the plasma membrane and result in a transient increase in intracellular $\mathrm{Ca}^{2+}$. In soybeans, the elevation of cytosolic $\mathrm{Ca}^{2+}$ leads to the activation of calmodulins, which play roles in signaling to activate defense-related genes (Heo et al. 1999). The victorin-induced expression of AsHHT1 and AsCCoAOMT genes was blocked by trifluoperazine, suggesting that calmodulin may also be a signal mediator, at least for defense-related genes in oats.

CCoAOMT was proposed to be involved in plant defense reactions (Matern et al. 1995; Pakusch et al. 1989). FeruloylCoA, the product of CCoAOMT, is used not only for phytoalexin biosynthesis but may also participate in cell wall reinforcement in oats. We demonstrated that a large amount of ferulic acid was cross-linked and esterified in cell walls at the infection sites in oats (Ikegawa et al. 1996), and CoA esters were defined as physiological cosubstrates in the esterification of polysaccharides for cell wall reinforcement (Meyer et al. 1991). Thus, the rapid accumulation of AsCCOAOMT transcripts in victorin-treated $P c-2 / V b$ oat lines (Fig. 6) supports the interpretation that victorin functions as a specific elicitor of resistance expression in $P c-2 / V b$ oats and not as a classic toxin that simply damages cells (Litzenberger 1949; Mayama et al. 1986). Transforming oat plants with sense or antisense constructs of AsCCOAOMT and AsHHT genes may allow a closer evaluation of the role of avenanthramidesuse and provide new strategies to control disease, as was illustrated with the resveratrol synthase gene (Hammerschmidt 1999).

\section{MATERIALS AND METHODS}

\section{Plant materials and elicitor treatment.}

Four cultivars of oat, A. sativa cvs. Shokan 1, PC-38, Iowa×469, and Iowa×424 were used in this study. 'Shokan 1' is highly resistant to race 226 but susceptible to race 203 of the oat crown rust fungus. Conversely, 'PC-38' is resistant to race 203 but susceptible to race 226 . 'Iowa 469 ' carries the $P c 2 / V b$ gene, which confers specific susceptibility to the Victoria blight fungus $C$. victoriae and also specific sensitivity to victorin $\mathrm{C}$, a host-selective phytotoxin produced by this fungus (Mayama et al. 1995a; Wolpert et al. 1985). 'Iowax424', a near-isogenic line of 'Iowax469', does not carry the $P c-2 / V b$ gene and is insensitive to victorin $C$. The oat seeds were soaked in water for $2 \mathrm{~h}$, were sown in vermiculite, and were grown in a growth chamber at $20^{\circ} \mathrm{C}$ for $16 \mathrm{~h}$ at $10,000 \mathrm{Lux}$ daily. Seven-day-old primary leaves were used for all experiments and were treated with various elicitors as described previously (Tada et al. 2001). The effect of elicitors on the induction of phytoalexin accumulation in oat was estimated after 24 $\mathrm{h}$ of treatment, by detecting the amount of avenanthramides in the solution with high-pressure liquid chromatography (Tada et al. 2001). Elicitors used for phytoalexin production included victorin C produced by $C$. victoriae (Wolpert et al. 1985), chitin-hexamers (hexa- $N$-acetylchito-oligosaccharides) and chitosan-hexamer purchased from Seikagaku Kogyo (Tokyo), and a crude extract of elicitors from Pseudomonas fluorescens (discussed below). Inhibitors were treated as described previously (Tada et al. 2001).

Inoculation of oat leaves with crown rust fungi was performed as described (Ikegawa et al. 1996). Uredospores of crown rust fungi were produced on susceptible hosts, and 10 $\mathrm{mg}$ of uredospores mixed with $90 \mathrm{mg}$ of talc were sprayed on approximately 100 primary leaves with a hand sprayer. Inoculated seedlings were incubated in a humid chamber in the dark for $16 \mathrm{~h}$ at $20^{\circ} \mathrm{C}$ and, then, were transferred to a controlled environmental growth chamber.

The plant growth-promoting rhizobacterium strain Pseudomonas fluorescens FPT 9601 (Aino et al. 1997) was grown at $30^{\circ} \mathrm{C}$ in Luria-Bertani (LB) medium $(100 \mathrm{ml})$ with $100 \mu \mathrm{g}$ of ampicillin per $\mathrm{ml}$ overnight and were pelleted by centrifugation at $5,000 \times g$ for $10 \mathrm{~min}$. The bacterial pellet was washed twice 
with 5 vol of $10 \mathrm{mM}$ sodium phosphate buffer ( $\mathrm{pH}$ 6.5) by repeated resuspension and centrifugation and, then, was resuspended in $2 \mathrm{vol}$ of $10 \mathrm{mM}$ sodium phosphate buffer ( $\mathrm{pH} \mathrm{6.5)}$. The bacterial suspension was heated in boiling water for $3 \mathrm{~min}$ and was centrifuged at $15,000 \times g$ for $10 \mathrm{~min}$. The 100 -fold dilution of the supernatant was used and was designated as FPT elicitor.

\section{Isolation of RNA and construction of a cDNA library.}

Total RNA from elicitor-treated oat leaves was isolated with the RNeasy plant minikit (Qiagen, Tokyo), according to the manufacturer's protocol. Poly (A) ${ }^{+}$RNA was isolated from oat leaves treated with FPT elicitor, using the PolyAT Tract System 1,000 mRNA kit (Promega, Tokyo) and, then, was used for cDNA synthesis with the ZAP cDNA synthesis kit (Stratagene, La Jolla, CA, U.S.A.). The cDNA was fractionated with Sephacryl S-500 and was extended with EcoRI adapters prior to ligation into the Uni-ZAP XR vector (Stratagene). The phagemids were packaged with ZAP-cDNA Gigapack III (Stratagene).

\section{cDNA probes.}

The Subtractor kit (Invitrogen Corporation, Carlsbad, CA, U.S.A.) was used to prepare subtracted cDNA. Briefly, first strand cDNA, which originated from oat leaves treated with FPT elicitor for 2 to $6 \mathrm{~h}$, was hybridized to an excess of photobiotinylated mRNA from uninduced oat leaves. The biotinylated mRNA cDNA was coupled with streptavidin and was removed by phenol-chloroform extraction. The unhybridized cDNAs were labeled with $\alpha-{ }^{32} \mathrm{P}-\mathrm{dCTP}(3,000 \mathrm{Ci} / \mathrm{mmol}$; Amersham Pharmacia Biotech, Tokyo), using a random primer labeling kit (Stratagene) and served as subtracted cDNA probes for screening of a cDNA library.

Oligonucleotide primers were synthesized by Amersham Pharmacia Biotech. mRNA isolated from FPT elicitor-treated oat leaves was used as template for RT-PCR amplification. RTPCR was performed according to standard methods (Sambrook et al. 1989), and annealing was done at $50^{\circ} \mathrm{C}$ for $20 \mathrm{~s}$.

\section{cDNA cloning and sequencing.}

A cDNA library of $1 \times 10^{5}$ recombinants was screened by in situ plaque-hybridization with a labeled subtracted cDNA probe or a HHT-specific cDNA probe. The hybridization was performed at $65^{\circ} \mathrm{C}$ for $16 \mathrm{~h}$ in $6 \times \mathrm{SSC}(1 \times \mathrm{SSC}$ is $0.15 \mathrm{M}$ $\mathrm{NaCl}$ plus $0.015 \mathrm{M}$ sodium citrate), $5 \times$ Denhardt's solution, $0.5 \%$ sodium dodecyl sulfate (SDS), and $100 \mu \mathrm{g}$ of heat-denatured salmon sperm DNA per ml. The filters were washed three times with $2 \times \mathrm{SSC}$ and $0.1 \% \mathrm{SDS}$ at $65^{\circ} \mathrm{C}$ for $30 \mathrm{~min}$ (Sambrook et al. 1989). Hybridizing phages were plaquepurified through two further rounds of screening. The cDNA inserts were rescued in pBluescript following the in vivo excision protocol for $\lambda$ ZAP (Stratagene), and the nucleotide sequence was determined with the Bigdye Terminator ready reaction kit and ABI Prism 310 genetic analyzer (PerkinElmer Corp., Norwalk, CT, U.S.A.).

\section{Heterologous enzyme expression.}

An NdeI restriction site was introduced at the start of translation, using a synthetic oligonucleotide primer 5'-GA GAA GAG CAT ATG AAG ATC ACG GT-3', which binds to nucleotides 125 to 144 of the AsHHT1 cDNA. Similarly, a BamHI restriction site was introduced just downstream of the translational terminator with another synthetic primer, 5'-C ATT GCT GGA TCC TAG TAA GCT GTA ATC-3', which was complementary to nucleotides 1,548 to 1,575 in the AsHHT1 cDNA. After amplification by PCR with the primers, modified AsHHT1 cDNA was digested with NdeI and BamHI. The di- gest was ligated with pET-22b (Novagen, Madison, WI, U.S.A.), yielding the plasmid pET-AsHHT1. Expression of HHT1 in $E$. coli BL21(DE3) (Novagen) was induced by the addition of 1 mM IPTG to cultures ( $\mathrm{A}_{600}$ of 0.3 to 0.4 ), and the cells were incubated for an additional $3 \mathrm{~h}$ at $37^{\circ} \mathrm{C}$, prior to harvesting by centrifugation at $5,000 \times g$ for $10 \mathrm{~min}$. The bacterial pellet was resuspended in buffer $(50 \mathrm{mM}$ Tris/Cl, pH 8.0, $2 \mathrm{mM}$ EDTA) \and lysed by freeze-thawing, and the lysate was subsequently cleared by centrifugation at $15,000 \times g$ for $5 \mathrm{~min}$. Protein expression was monitored by SDS-polyacrylamide gel electrophoresis. The catalytic activity of the protein was determined as described previously (Ishihara et al. 1997).

\section{Northern hybridization.}

Total RNA $(10 \mu \mathrm{g})$ from oat leaves was separated by electrophoresis on a $1.5 \%$ formaldehyde/agarose gel and blotted onto Hybond $\mathrm{N}^{+}$membranes. The blots were prehybridized for $4 \mathrm{~h}$ at $65^{\circ} \mathrm{C}$, followed by hybridization with a labeled probe for an additional $16 \mathrm{~h}$ at $65^{\circ} \mathrm{C}$, as described above (Sambrook et al. 1989). The membranes were subsequently washed three times with $2 \times \mathrm{SSC}$ and $0.1 \% \mathrm{SDS}$ at $65^{\circ} \mathrm{C}$ for $30 \mathrm{~min}$ and were packed with Saran Wrap for autoradiography.

\section{ACKNOWLEDGMENTS}

We thank the late N. T. Keen (University of California, Riverside, U.S.A.) for valuable suggestions. This work was supported by a Japan Society for the Promotion of Science postdoctoral fellowship for foreign researcher (to Q. Yang), special coordination funds for promoting science and technology, and by a grant-in-aid for scientific research (No. 12052215) from the Japanese Ministry of Education, Culture, Sports, Science and Technology.

\section{LITERATURE CITED}

Aino, M., Maekawa, Y., Mayama, S., and Kato, H. 1997. Biocontrol of bacterial wilt of tomato by producing seedlings colonized with endophytic antagonistic Pseudomonads. Pages 120-123 in: Proceedings of the 4th International Workshop on PGPR. A. Ogoshi, K. Kobayashi, Y. Homma, F. Kodama, N. Kondo, and S. Akino, eds. Nakanishi Printing, Sapporo, Japan.

Busam, G., Grimmig, B., Kneusel, R. E., and Matern, U. 1997. Isolation of tobacco cDNAs encoding caffeoyl-CoA 3-O-methyltransferase. Plant Physiol. 113:1003.

Busam, G., Junghanns, K. T., Kneusel, R. E., Kassemeyer, H. H., and Matern, U. 1998. Characterization and expression of caffeoyl-CoA 3$O$-methyltransferase in the induced resistance response of Vitis vinifera L. Plant Physiol. 115:1039-48.

Civardi, L., Rigau, J., and Puigdomenech, P. 1999. Nucleotide sequence of two cDNAs coding for caffeoyl-coenzyme A $O$-methyltransferase (CCoAOMT) and study of their expression in Zea mays. Plant Physiol. 120:1206.

Dixon, R. A., and Paiva, N. L. 1995. Stress induced phenylpropanoid metabolism. Plant Cell 7:1085-1097.

Douglas, C. J. 1996. Phenylpropanoid metabolism and lignin biosynthesis: From weeds to trees. Trends Plant Sci. 1:171-178.

Grayer, R. J., and Kokubun, T. 2001. Plant-fungal interactions: The search for phytoalexins and other antifungal compounds from higher plants. Phytochem. 56:253-263.

Hahlbrock, K., and Scheel, D. 1989. Physiology and molecular biology of phenylpropanoid metabolism. Ann. Rev. Plant Physiol. 40:347-369.

Hammerschmidt, R. 1999. Phytoalexins: What have we learned after 60 years? Ann. Rev. Phytopathol. 37:285-306.

Heo, W. D., Lee, S. H., Kim, J. C., Chung, W. S., Chun, H. J., Lee, K. J., Park, C. Y., Park, H. C., Choi, J. Y., and Cho, M. J. 1999. Involvement of specific calmodulin isoforms in salicylic acid-independent activation of plant disease resistance responses. Proc. Natl. Acad. Sci. U.S.A. 96:766-771.

Ikegawa, T., Mayama, S., Nakayashiki, H., and Kato, H. 1996. Accumulation of diferulic acid during the hypersensitive response in oat leaves and it's role in enhanced resistance to cell-wall degrading enzyme. Physiol. Mol. Plant Pathol. 48:245-255.

Inoue, K., Sewalt, V. J. H., Ballance, G. M., Ni, W., Sturzer, C., and Dixon, R. A. 1998. Developmental expression and substrate specifici- 
ties of alfalfa caffeic acid 3-O-methyltransferase and caffeoyl coenzyme A 3-O-methyltransferase in relation to lignification. Plant Physiol. 117:761-770.

Ishihara, A., Matsukawa, T., Miyagawa, H., Ueno, T., Mayama, S., and Iwamura, H. 1997. Induction of hydroxycinnamoylCoA:hydroxyanthranilate $N$-hydroxycinnamoyl-transferase (HHT) activity in oat leaves by victorin C. Z. Naturforsch. 52c:756-760.

Ishihara, A., Miyagawa, H., Kuwahara, Y., Ueno, T., and Mayama, S. 1996. Involvement of $\mathrm{Ca}^{2+}$ ion in phytoalexin induction in oats. Plant Sci. 115:9-16.

Ishihara, A., Ohtsu, Y., and Iwamura, H. 1999. Biosynthesis of oat avenanthramide phytoalexins. Phytochem. 50:237-242.

Litzenberger, S. C. 1949. Nature of susceptibility to Helminthosporium victoriae and resistance to Puccinia coronata in Victoria oats. Phytopathol. 39:300-318.

Luke, H. H., Murphy, H. C. And Peter, F. C. 1966. Inheritance of spontaneous mutations of the Victoria locus in oats. Phytopathol. 56:210-212.

Matern, U., Grimmig, B., and Kneusel, R. E. 1995. Plant cell wall reinforcement in the disease resistance response: Molecular composition and regulation. Can. J. Bot. 73:511-517.

Mayama, S., Tani, T., and Matsuura, Y. 1981. The production of phytoalexins by oat in response to crown rust, Puccinia coronata f. sp. avenae. Physiol. Plant Pathol. 19:217-226.

Mayama, S., Matsuura, Y., Iida, H., and Tani, T. 1982. The role of avenlumin in the resistance of oat to crown rust, Puccinia coronata f. sp. avenae. Physiol. Plant Pathol. 20:189-199.

Mayama, S., Tani, T., Midland, S. L., Sims, J. J., and Keen, N. T. 1986. The purification of victorin and its phytoalexin elicitor activity in oat leaves. Physiol. Mol. Plant Pathol. 29:1-18.

Mayama, S., Bordin, A. P. A., Morikawa, T., Tanpo, H., and Kato, H. 1995a. Association between avenalumin accumulation, infection hypha length and infection type in oat crosses segregation for resistance to Puccinia coronata f. sp. avenae race 226. Physiol. Mol. Plant Pathol. 46:255-261.

Mayama, S., Bordin, A. P. A., Morikawa, T., Tanpo, H., and Kato, H. 1995b. Association of avenalumin accumulation with co-segregation of victorin sensitivity and crown rust resistance in oat lines carrying the Pc-2 gene. Physiol. Mol. Plant Pathol. 46:263-274.

Mayama, S., Tada, Y., Hata, S., Takata, Y., Yao, N., Mori, T., Yang, Q., Betsuyaku, S., Nakayashiki, H., Tosa, Y., and Park, P. 2001. Apoptotic response in defense of oats to infections and elicitors. Pages 220-228 in: Delivery and Perception of Pathogen Signals to Plants. N. T. Keen, S. Mayama, J. E. Leach, and S. Tsuyumu, eds. American Phytopathological Society, St. Paul, MN, U.S.A

Meyer, K., Kohler, A., and Kauss, H. 1991. Biosynthesis of ferulic acid esters of plant cell wall polysaccharides in endomembranes from parsley cells. FEBS (Fed. Eur. Biochem. Soc.) Lett. 290:209-212.

Miyagawa, H., Ishihara, A., Nishimoto, T., Ueno, T., and Mayama, S. 1995. Induction of avenanthramides in oat leaves inoculated with crown rust fungus, Puccinia coronata f. sp. avenae. Biosci. Biotech. Biochem. 59:2305-2306.

Miyagawa, H., Ishihara, A., Kuwahara, Y., Ueno, T., and Mayama, S. 1996. Comparative studies of elicitors that induce phytoalexin in oats. J. Pestic. Sci. 21:203-207.

Niemann, G. J. 1993. The anthranilamide phytoalexins of the Caryophyllaceae and related compounds. Phytochem. 34:319-328.

Niemann, G. J. 1994. A crucial role of phenolic metabolism in resistance of carnations to wilt diseases. Acta Horticult. 381:565-571.

Pakusch, A. E., Kneusel, R. E., and Matern, U. 1989. S-Adenosyl-Lmethionine: Trans-caffeoyl-coenzyme A 3-O-methyltransferase from elicitor-treated parsley cell suspension cultures. Arch. Biochem. Biophysics 271:488-494.

Reinhard, K., and Matern, U. 1989. The biosynthesis of phytoalexins in Dianthus caryophyllus L. cell cultures: Induction of benzoylCoA:anthranilate $N$-benzoyltransferase activity. Arch. Biochem. Biophys. 275:295-301.

Sambrook, J., Fritsch, E. P., and Maniatis, T. 1989. Molecular Cloning: A Laboratory Manual. Cold Spring Habor Laboratory Press, Cold Spring Harbor, NY, U.S.A.

Schmitt, D., Pakusch, A. E., and Matern, U. 1991. Molecular cloning, induction and taxonomic distribution of caffeoyl-coenzyme A 3-O methyltranserase, an enzyme involved in disease resistance. J. Biol. Chem. 266:17416-17423.

Tada, Y., Hata, S., Nakayashiki, H., Tosa, Y., and Mayama, S. 2000. Signal mediators for phytoalexin production in defense response of oats elicited by victorin as a specific elicitor. J. Gen. Plant Pathol. 66:185-190.

Tada, Y., Hata, S., Takata, Y., Nakayashiki, H., Tosa, Y., and Mayama, S. 2001. Induction and signaling and apoptotic response typified by DNA laddering in the defense response of oats to infection and elicitors. Mol. Plant-Microbe Interact. 14:477-486.

Wolpert, T. J., Macko, V., Acklin, W., Jaun, B., Seibl., J., Meili, J., and Arigoni, D. 1985. Structure of victorin C, the major host-selective toxin from Cochliobolus victoriae. Experientia 41:15241529.

Yang, Q., Reinhard, K., Schiltz, E., and Matern, U. 1997. Characterization and heterologous expression of hydroxycinnamoyl benzoyl-CoA:anthranilate $N$-hydroxy-cinnamoyl/benzoyl transferase from elicited cell cultures of carnation, Dianthus caryophyllus L. Plant Mol. Biol. 35:777-789.

Yao, N., Tada, Y., Park, P., Nakayashiki, H., Tosa, Y., and Mayama, S. 2001. Novel evidence for apoptotic cell response and differential signals in chromatin condensation and DNA cleavage in victorin-treated oats. Plant J. 28:13-26.

Yao, N., Imai, S., Tada, Y., Nakayashiki, H., Tosa, Y., Park, P., and Mayama, S. 2002. Apoptotic cell death is a common response to pathogen attack in oats. Mol. Plant-Microbe Interact. 15:1000-1007.

Ye, Z.-H., Kneusel, R. E., Matern, U., and Varner, J. E. 1994. An alternative methylation pathway in lignin biosynthesis in Zinnia. Plant Cell 6:1427-1439. 